\title{
SÍNCOPE CONVULSIVO COMO PRIMERA MANIFESTACIÓN CLÍNICA DE PARAGANGLIOMA CAROTÍDEO
}

\author{
Laura Barreto MD*, Eduardo Palacios MD**
}

\section{Resumen}

El síncope convulsivo se define como episodio de pérdida de la conciencia con postura tónica. Hace parte de los diagnósticos diferenciales de la epilepsia, como evento paroxístico con manifestaciones motoras, clónica y/o mioclónica, sin descargas epileptiformes en el electroencefalograma. La crisis del síncope hace parte de la respuesta del cerebro frente a estados de hipoxia y representa un reto que ha generado controversia acerca del comportamiento y conducta a seguir. Se reporta el caso de un hombre de 53 años que ingresa a urgencias por presentar un único episodio sincopal, asociado con postura tónica en extensión de extremidades superiores, supraversión de la mirada y relajación de esfínteres. Los estudios de extensión mostraron una masa dependiente de la carótida izquierda en su bifurcación compatible con paraganglioma, que a la palpación generaba la aparición de eventos similares al del ingreso simulando semiología ictal.

Palabras clave: síncope convulsivo, paraganglioma.

\section{CONVULSIVE SYNCOPE AS FIRST CLINICAL MANIFESTATION OF CAROTID PARAGANGLIOMA}

\section{Abstract}

Convulsive syncope is defined as an episode of loss of consciousness and tonic posture. It is part of epilepsy's differential diagnoses, as a paroxysmal event with motor clonic and/or myoclonic activity, showing no epileptiform discharges in the electroencephalogram. Syncope episodes are a component of the brain's response to hypoxia and represent a challenge which remains controversial on adequate handling and therapy. We report on a 53-year old male patient who presented to the emergency room for having experienced one syncope episode associated with tonic postures involving extension of the upper extremities, upward gaze and urine and fecal incontinence. The diagnostic work up revealed a mass in the left common carotid artery bifurcation compatible with a paraganglioma. Similar events to that of admission were triggered by palpation of the mass simulating ictal semiology.

Key words: convulsive syncope, paraganglioma.

Fecha recibido: julio 30 de 2012 - Fecha aceptado: diciembre 10 de 2012

* Residente II de Neurología, Fundación Universitaria de Ciencias de la Salud. Bogotá DC, Colombia.
** Jefe del Servicio de Neurología, Hospital de San José. Profesor Titular, Fundación Universitaria de Ciencias de la Salud. Miembro de la Sociedad de Cirugía de Bogotá DC, Colombia. 


\section{Caso clínico}

Paciente de sexo masculino de 53 años de edad que ingresa al servicio de urgencias por cuadro clínico de inicio súbito con sensación de debilidad generalizada, náusea y diaforesis profusa; refiere ver imagen de un rostro familiar después de tomar postura en bipedestación.

Minutos después presenta ruptura de contacto con el entorno, pérdida del tono postural, versión cefálica hacia la derecha, supraversión de la mirada y postura tónica en extensión de extremidades superiores, asociada con movimientos clónicos y relajación de esfínter vesical. Este episodio dura cerca de tres minutos con recuperación gradual y espontánea de la conciencia y luego múltiples episodios de emesis, razón por la que deciden trasladarlo a urgencias.

No hay antecedentes personales patológicos de importancia. Al examen físico de ingreso llama la atención la presencia de una masa indurada no pulsátil adherida a planos profundos en la zona II de cuello, que a la palpación desencadena cambios disautonómicos con síntomas de náusea, diaforesis, ilusión rotacional de objetos estáticos y descenso de la presión arterial y la frecuencia cardíaca. Minutos después se evidencia ruptura de contacto con el entorno, suspensión momentánea del lenguaje, automatismos orolinguales y mandibulares, midriasis y adquiere una postura tónica con extensión de extremidades superiores y movimientos clónicos. El episodio dura cerca de tres minutos, con posterior estado de somnolencia y recuperación gradual de los signos vitales que se acompaña de una mejoría del estado de conciencia.

Dentro de los estudios de extensión realizados se encuentra en el Doppler una imagen sugestiva de paraganglioma ubicado en la bifurcación de la carótida común izquierda, con diámetros aproximados de 35 x 35 mm (Figuras 1 y 2).

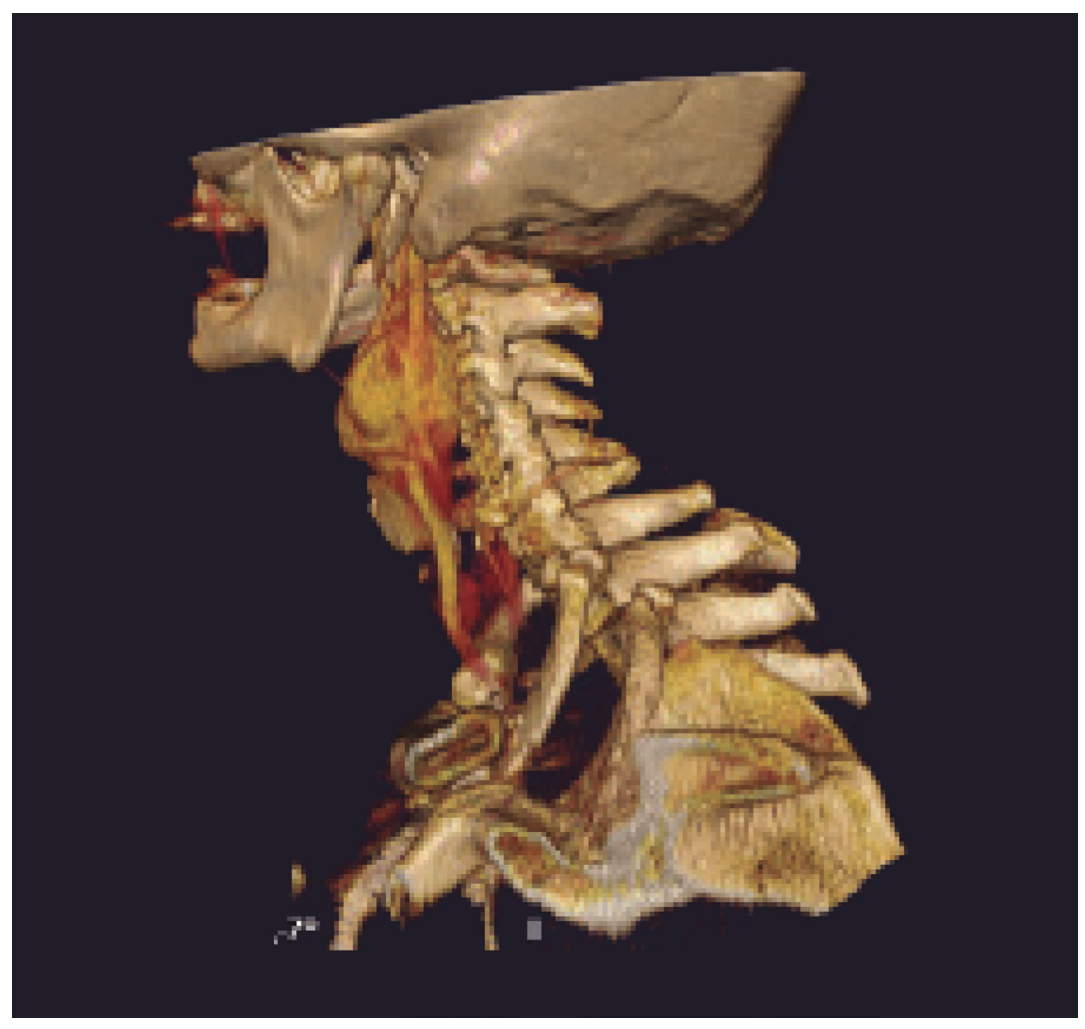

Figura I. Reconstrucción tridimensional, angiotac vasos del cuello. 


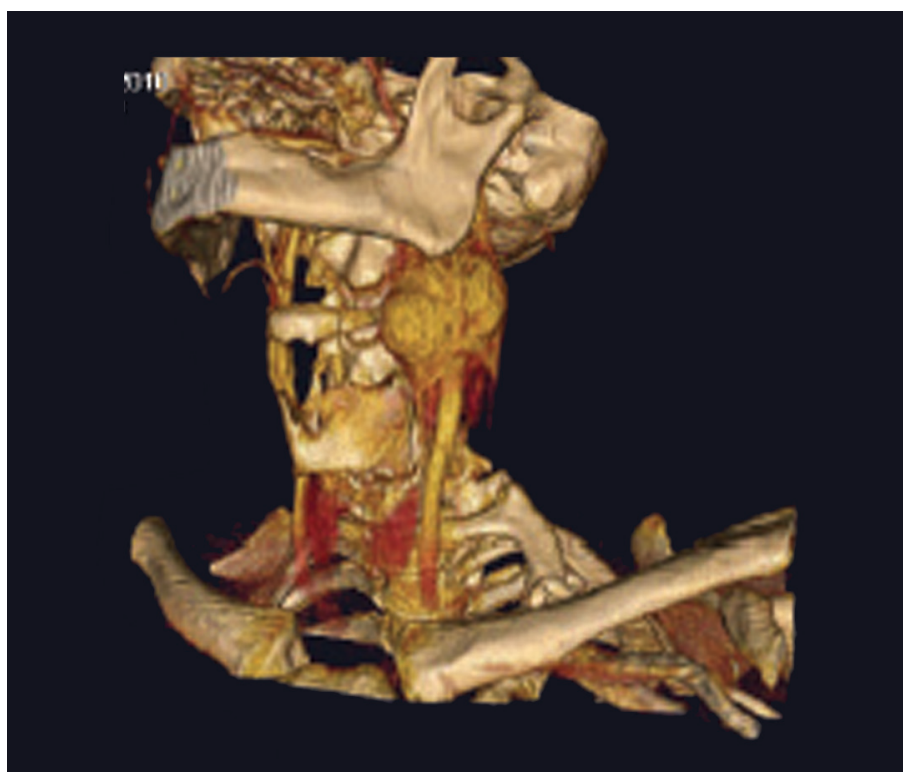

Figura 2. Reconstrucción tridimensional.

Se decide ampliar el estudio mediante monitorización electroencefalográfica con video (videotelemetría) para determinar diagnósticos diferenciales de crisis epilépticas, registrando la actividad cerebral durante los episodios y las manifestaciones motoras desencadenados al palpar la masa del cuello. No se registró actividad epileptiforme y solo hubo evidencia de actividad lenta difusa. Ante estos hallazgos se considera que el paciente cursa con eventos paroxísticos no epilépticos de tipo síncope convulsivo, con episodios desencadenados al manipular la masa en cuello o realizar rotación cefálica, lo cual causa síntomas y signos de bajo gasto que sugieren paraganglioma productor intermedio de catecolaminas. Es intervenido quirúrgicamente para resección del tumor, cuyo reporte de patología demostró un paraglanglioma carotídeo productor intermedio de catecolaminas. Después de la cirugía desaparecieron los episodios y al paciente se da egreso sin medicamentos antiepilépticos.

\section{Discusión}

En la fase inicial presenta signos prodrómicos de síncope vasovagal, pero por el comportamiento del episodio se propone el diagnóstico diferencial de crisis epilépticas sintomáticas, dadas las características clínicas que sugieren semiología ictal.
No hay indicios de que las convulsiones sincopales reflejen actividad epiléptica de la corteza cerebral. Por el contrario, la actividad muscular durante el evento es subcortical y se origina a partir de una activación anormal de la formación reticular del tallo cerebral, como consecuencia de la hipoxia de las neuronas reticulares mediada por quimiorreceptores y la liberación de la inhibición cortical. ${ }^{1}$

En el electroencefalograma del síncope, sea convulsivo o no, aparece una secuencia de ondas lentas generalizadas de elevada amplitud y el regreso de ondas lentas. La actividad de fondo es normal y refleja la manifestación de hipoxia cerebral; la pérdida del conocimiento se relaciona con la aparición de actividad de ondas lentas, mientras que las crisis epilépticas representan descargas en el trazado.

Dentro de las manifestaciones clínicas del síncope, el $10 \%$ corresponde a convulsiones que se acompañan con síntomas prodrómicos como náusea, distorsión de la realidad, alucinaciones visuales y diaforesis. Las mioclonias segmentarias multifocales asincrónicas lo diferencian de las epilépticas que se comportan de manera simétrica con menor tiempo de duración. ${ }^{2}$

La actividad tónica se encuentra con mayor frecuencia en pacientes con síncopes por asistolia en donde 
hay una interrupción total del flujo cerebral, con compromiso de la cabeza o bien extensión o flexión de extremidades superiores, que pueden acompañarse de automatismos como lamerse los labios, masticación o giros cefálicos. La mayoría de estos episodios son de corta duración y repetitivos, los ojos pueden permanecer abiertos con desviación de la mirada, las alucinaciones ya sean en síncope convulsivo o no, aparecen hasta en el $60 \%$ de los casos, en la mayoría visuales y auditivas, con luces destellantes e imágenes estructuradas o de rostros familiares, además de percepción de melodías o sonidos fuertes. ${ }^{1}$

Dentro de las manifestaciones previas al síncope se describen tinutus, síntomas vegetativos de corta duración y curvas descritas como autonómicas en las que se preserva la conciencia y por lo general son de mayor tiempo de duración que las que preceden las crisis epilépticas del lóbulo temporal, con epigastralgia o percepción de olores o sabores. ${ }^{3}$

Es importante conocer los fenómenos posictales y los de estos eventos, ya que son parte clave para la diferenciación entre el síncope y las crisis epilépticas. En el primero la recuperación de la conciencia es espontánea e inmediata con máxima duración de 30 segundos, a diferencia de las crisis epilépticas en donde es más prolongada y se asocia con estado de desorientación en tiempo y espacio. En las dos entidades se puede presentar relajación de esfínteres.

Las crisis epilépticas ocurren casi siempre en forma espontánea, mientras que el síncope es provocado por acciones o circunstancias específicas. Los automa- tismos que aparecen en estas dos entidades pueden simular crisis parciales complejas, sin embargo es importante reconocer el comportamiento posterior ya que en su gran mayoría los epilépticos se acompañan de fenómeno posictal, son de más larga duración y con mayor compromiso del estado de conciencia. ${ }^{3}$

El reconocimiento y adecuada diferenciación entre estas dos entidades generan una especificidad y sensibilidad de $94 \%$, que influyen de manera importante en la intervención y manejo médico posterior. Es importante realizar estudio con electrocardiograma y electroencefalograma a todos los pacientes en los que la semiología del episodio no sugiere patrón específico que oriente hacia una de las dos condiciones.

Cabe resaltar el papel que desempeñan los marcadores bioquímicos y su comportamiento en cada una de estas entidades clínicas, como lo son CPK total y prolactina, que pueden tener un patrón de positividad en las fases agudas pero no representan un marcador de confianza para establecer con seguridad el diagnóstico, ya que pueden elevarse en cualquiera de las dos.

\section{Referencias}

1. Continuum Lifelong Learning Neurol 2010; 16 (3): 36-56.

2. Brenner RP. Elecrtroencephalography in syncope. J Clin Neurophysiol 1997; 14 197-209. Revista española de cardiología Journal Of the American College of Cardiology Vol. 40 No. 1, 2002@ 2002 by the American College of Cardiology Foundation ISSN 0735-1097/02/\$22.00 Published by Elsevier Science Inc. PII S0735-1097(02)01940-X.

3. Instituto Nacional de Oncología y Radiobiología. Quemodectomas de región cervicofacial. Rev. Cubana Medicina 2008. 